\title{
Pathophysiology of Early Vascular Ageing-Opportunities for Treatment
}

\author{
Vasilios Kotsis ${ }^{1, *}$, Christina Antza ${ }^{1}$ and Stella Stabouli ${ }^{1,2}$ \\ ${ }^{I}$ Hypertension Center, $3^{\text {rd }}$ Department of Medicine, Papageorgiou Hospital, Aristotle University of Thessaloniki, \\ Greece; ${ }^{2} I^{\text {st }}$ Department of Pediatrics, Hippokration Hospital, Aristotle University Thessaloniki, Greece
}

\begin{abstract}
The story of vascular ageing was first described in the seventeenth century when Thomas Sydenham wrote that 'A man is as old as his arteries'. This aphorism was returned to publicity lately when Peter Nilsson reintroduced the concept of early vascular ageing. As vascular ageing is described a gradual process involving biochemical, enzymatic, and cellular changes of the vasculature and modification of the signals that modulate them. In susceptible individuals this process appears to be accelerated, leading to features that comprise a condition characterized as early vascular aging (EVA). Early vascular ageing represents the acceleration of the vascular ageing process.
\end{abstract}

Keywords: Arterial stiffness, ageing, early vascular ageing.

\section{INTRODUCTION}

The incidence of cardiovascular diseases such as stroke, hypertension, and coronary heart disease is increasing over the years and is more common in the elderly. The story of vascular ageing was first described in the seventeenth century when Thomas Sydenham wrote that 'A man is as old as his arteries' [1]. This aphorism was returned to publicity lately when Nilsson et al. [2] reintroduced the concept of early vascular ageing. Aging of the aorta and elastic arteries causes arterial stiffening and leads to development of cardiac failure and microvascular disease in highly perfused organs such as the brain and kidneys and describe aspects of the socalled cardiovascular ageing continuum [2,3]. It has also been observed that vascular alterations that occur in apparently otherwise healthy senior people, including an increase in stiffness and thickness of large arteries as well as endothelial dysfunction, seem to be more extensive in patients with hypertension or atherosclerosis at an earlier age [4]. Based on this research it seems that apart from age, important factors of vascular ageing are the classical cardiovascular risk factors.

As vascular ageing is described a gradual process involving biochemical, enzymatic, and cellular changes of the vasculature and modification of the signals that modulate them [5]. In susceptible individuals this process appears to be accelerated, leading to features that comprise a condition characterized as early vascular ageing (EVA). Early vascular ageing represents the acceleration of the vascular ageing process. Aspects of this premature process constitute the increased arterial stiffness, increased intima-media thickness, impaired dilation of central elastic arteries, and impaired endothelial function. In this review we are going to analyze possible pathophysiological mechanisms of early vascular ageing and the relationship of arterial stiffness to cardiovascular events. The improvement of cardiovascular risk

\footnotetext{
*Address correspondence to this author at the Hypertension-ABPM Center Papageorgiou Hospital, Zaka 39, Panorama 55236, Thessaloniki, Greece; Tel: +30 6974748860; Fax: +30 2310452429; E-mail: vkotsis@auth.gr
}

through proper medication is also going to be discussed in our effort to impose the normal ageing process acting against knowing aggravating and accelerating factors.

\section{PATHOPHYSIOLOGY OF VASCULAR AGEING AND EARLY VASCULAR AGEING}

Ageing and vascular ageing are thought to share common underlying molecular and cellular mechanisms, including those leading to oxidative damage of macromolecules and organelles such as mitochondria, formation of advanced glycation end products, telomere damage, depletion of vascular progenitor cells and the accumulation of senescent in the endothelial and vascular smooth muscle cells.

Specifically vascular ageing is characterized by endothelial dysfunction (resulting primarily from a reduction in nitric oxide bioavailability), diffuse intimal thickening, arterial wall stiffening, vascular calcification and defective vascular repair [6]. In susceptible individuals this process seem to be accelerated in the presence of cardiovascular risk factors leading to a number of features that are integrated in a condition characterized as EVA [7]. At first endothelial cell activity seems to play a significant role in regulating central arterial stiffness possibly via intercellular adhesion molecule-1, transforming growth factor (TGF)-b and nicotinamide adenine dinucleotide phosphate-oxidase increase with parallel decrease in vascular endothelial growth factor (VEGF) and nitric oxide bioavailability. However, this hypothesis has only been proved in animal models $[8,9]$.

Another interesting hypothesis is the role of a shorter telomere length on vascular aging. Studies have described that increased chronological age is associated with a decrease in telomerase reverse transcriptase in arterial endothelial cells. This enzyme is responsible for restoring the decreased length of telomeres at the end of every cellular replication. Decreased transcriptase activity has been thought to be important for increased vascular aging [10-14]. Cross-sectional studies have also reported that subjects with increased arterial stiffness have shorter telomeres [15]. Furthermore, athe- 
rosclerosis and its preclinical status of plaque formation and intima-media thickening of the arterial wall have been proven to have an exacerbating effect on the aging process $[16,17]$.

Importantly, these processes are accentuated in the presence of classical cardiovascular risk factors and in vascular pathologies. In particular, most of these molecular and/or cellular events are exacerbated in the context of diabetes and the metabolic syndrome, which in turn are also strongly associated with endothelial dysfunction and with vascular stiffness and thickness [18]. However, even though the existence of these associations has been widely documented, the relationships between risk factors (genetic and/or environmental), molecular/cellular events, age-related vascular changes and cardiovascular pathologies are not entirely understood. The classical risk factors only in part can explain the vascular ageing process and why it is accelerated in some patients. Moreover, animal studies have clearly shown that an increased short-term blood pressure variability might induce arteriosclerotic lesions in the arterial wall leading to a reduced arterial distensibility $[19,20]$. Interestingly in hypertensive humans increased systolic blood pressure is associated with increased intima-media thickness (IMT) of the carotid arteries [21]. Recently a study from our group in a population of young healthy volunteers showed that the variability of the 24-h systolic blood pressure was independently associated with arterial stiffness and early vascular ageing [22]. The increased shear stress of the large arteries such as aorta even in a pulsate cyclic way seems to be important in vascular ageing. Another possible mechanism through which hypertension speeds up the arterial aging process is the activation of the renin-angiotensin system. Induction of Angiotensin II (Ang II) in endothelial cells promoted a growth arrest with phenotypic characteristics of cell senescence, such as enlarged cell shapes, increased senescence-associated beta-galactosidase positive staining cell, and depressed cell proliferation. Apoptotic changes were increased in senescent cells, with a small subset of the senescent cells showing aberrant morphology such as pronounced nuclear fragmentation or multiple micronuclei [23]. Other possible mechanisms of arterial stiffening in hypertension are the relation of high blood pressure with increased sodium and the reduced potassium intake. High plasma sodium levels block NO synthesis and induce endothelial dysfunction through increased aldosterone, while high plasma potassium levels activate NO release [24].

A major characteristic of the immune system during aging is the up-regulation of the inflammatory responses which appears to be detrimental for longevity. Recently studies observed a progressive age-dependent increase of IL-2, IFNgamma, TNF-alpha, IL-4, IL-6, IL-10 positive CD8+ T-cells [25]. Chronic low-grade inflammation can exaggerate the normal ageing process regardless of age. These structural changes of the arterial wall may also be influenced by molecular and genetic factors $[25,26]$. Moreover, smoking can accelerate aging probably through the overproduction of the reactive oxidative species (ROS) during mitochondrial metabolic activity or by inhibiting the DNA repair function [2].

\section{ARTERIAL STIFFNESS}

Aging represents a major determinant of arterial stiffness. Histological studies have shown extensive impairment to the medial elastin fiber network on central elastic arteries, such as the proximal aorta and the carotid artery, while subtle aging alterations have been found in distal muscular arteries because of impaired distensibility [27]. Specifically arterial stiffness is increased when elastic properties of the arterial wall are reduced. Ageing is a procedure that causes structural and functional modifications in the vasculature, resulting in decreased arterial compliance and increased arterial stiffness $[28,29]$. Arterial stiffness is an ongoing procedure starting from the early years of life, advancing with age and modified from different factors [30]. At this point it is interesting to mention that arterial stiffness increases with age, hypertension, diabetes mellitus, atherosclerosis, and end-stage renal disease [31-35].

Cell-matrix interaction is a key player in the regulation of arterial stiffness [36]. This is supported by recent research in which genetic markers of arterial stiffness, one being Col4A1, associated with collagen synthesis on chromosome 13 [37], and another associated with a putative gene enhancer in the VRK1-BCL11B gene desert on chromosome 14 [38], are independent from the overall genetic architecture of hypertension and blood pressure regulation [39].

Carotid-femoral pulse wave velocity (PWV) has been considered as a direct measurement of arterial stiffness. Pulse wave velocity is the speed at which the pressure waveforms travel along the aorta and large arteries during each cardiac cycle. PWV has a better predictive value than classical cardiovascular risk factors possibly because it depicts the cumulative effect of both known and non-identified cardiovascular risk factors on the large arteries [40], whereas $\mathrm{BP}$, glucose and lipid levels may not, as they vary from period to period. Therefore, it represents a useful spectrum through which the EVA process could be thoroughly yet non-invasively investigated. Thus, arterial stiffness measurement can quantify the arterial impairment and classify a patient at higher risk level. Indeed, a strong and graded association between PWV and coronary heart disease and stroke has been found [41]. Also aortic PWV is a powerful independent predictor of mortality in both diabetes and impaired glucose tolerance population samples [42].

\section{ARTERIAL STIFFNESS AND EVA IN PARTICULAR MEDICAL INCIDENTS}

Typical examples of early vascular ageing are the following pathological conditions. Carotid stiffness significantly increased in patients with chronic kidney disease [43]. Patients with rheumatoid arthritis also reported to have increased arterial stiffness. The risk of acute myocardial infarction in rheumatoid arthritis patient corresponding to the risk seen in a patient 10 years older. These patients usually suffer from cardiovascular events at a younger age and with a higher mortality rate [44]. Moreover it has been recently suggested that an inflammatory process is likely, generated by neoantigens activating $\mathrm{T}$ cells. This initial response might lead to entry of effector-like $T$ cells into the perivascular fat with consequent release of cytokines and other inflammatory mediators, which in concert with the direct effects of angiotensin II and catecholamines cause vascular damage and remodeling [45]. It is also noteworthy that the reduction of inflammation through anti-TNF a medical therapy can improve arterial stiffness [43]. 
Blood pressure is the most important explanatory variable of arterial stiffening. Asmar et al. [46] showed that ambulatory blood pressure monitoring might provide a sensitive approach for evaluating the relationship between dysfunction of the large arteries and blood pressure in smokers. The study provided evidence that, in spite of similar casual blood pressure levels among smokers and non-smokers, smokers have a higher systolic and pulse pressure during exclusively during the activity daytime period suggesting a possible role of cyclic stress in the deterioration in the structure of the hypertensive arterial wall. $24 \mathrm{~h}$ pulse pressure and variability were also increased in subjects with carotid endarterectomy [47]. Pulse pressure is the difference between systolic and diastolic blood pressure. Age increases systolic blood pressure, while diastolic blood pressure is reduced in the elderly causing an increased pulse pressure. Increased pulse pressure is in many cases synonymous with increased arterial stiffness. Central pulse pressure is determined by the left ventricular ejection rate, the arterial distensibility and the arterial wave reflections $[48,49]$. Age reduces the compliance of the arterial tree and the reflected waves in the periphery return early in the systole in the aorta causing an increase in central systolic and pulse pressure.

\section{EARLY VASCULAR AGEING: A SUBSTRATE FOR CARDIOVASCULAR DISEASE}

Aortic stiffness expressed as aortic PWV is a strong predictor of future $\mathrm{CV}$ events and all-cause mortality. The predictive ability of arterial stiffness is higher in subjects with a higher baseline CV risk [50]. The concept of early vascular ageing is now widely accepted as inappropriate for age aortic stiffness, suggesting an increased risk for cardiovascular events independent of chronological age. For the moment, the only tools that are provided to estimate cardiovascular risk are based on integration of conventional risk factors [6]. However, it has been found that carotidfemoral PWV provides additional information for risk prediction of cardiovascular events [51]; therefore its use could be helpful for clinical screening of patients with early vascular ageing and re-stratification of cardiovascular risk beyond classical scores.

A study has shown that increased PWV values were significantly associated with cardiovascular disease mortality in a Japanese male population. Those patients who had PWV values $>9.0 \mathrm{~m} / \mathrm{s}$ had a higher risk of mortality from all causes of death, as well as cardiovascular-related death [52]. These results are reinforced by the Rotterdam Study with 2835 participating subjects. During follow-up, 101 subjects developed coronary heart disease (mean follow-up period, 4.1 years), and 63 subjects developed a stroke (mean follow-up period, 3.2 years). The risk of cardiovascular disease increased with increasing aortic pulse wave velocity index. The study indicates that aortic pulse wave velocity is an independent predictor of coronary heart disease and stroke in apparently healthy subjects [41]. Moreover, in patients with end-stage renal disease, increased aortic stiffness determined by measurement of aortic PWV is a strong independent predictor of all-cause and mainly cardiovascular mortality [53]. Also, another study in 397 diabetic patients reported that the patients who died were significantly older, smoked more, had increased PWVs by $2.6 \mathrm{~m} / \mathrm{sec}$ and increased mean sys- tolic pressures by $10 \mathrm{~mm} \mathrm{Hg}$ than those who survived. Then PWV and systolic pressure were compared. Those who died had higher baseline PWVs on average for any level of baseline systolic pressure levels [42].

\section{TREATMENT OF EVA}

Because of the inability of science to prevent natural aging we should put our hopes in therapeutic strategies designed to reverse the factors that affect and cause EVA. Lifestyle intervention is still the key-point for intervention directed toward EVA and cardiovascular risk. Nonpharmacological treatments are able to reduce arterial stiffness and have been detailed in several reviews and include additional dietary changes such as low salt diet, moderate alcohol consumption, as well as increased intake of garlic powder, a-linoleic acid, fish oil, green tea, and dark chocolate [54]. EVA patients could benefit for an early and nonexpensive intervention on vascular ageing based on physical exercise [55].

Pharmacological intervention can also reduce early vascular ageing. Several studies, provided evidence that antihypertensive agents may decrease early vascular ageing. Renin-angiotensin-aldosterone system blockers are effective agents in reducing arterial stiffness through inhibition of the vasoconstrictive effect of angiotensin II and improvement of endothelial function. Aldosterone blockers may also act similarly. Nitro-vasodilators reduce blood pressure and wave reflections because their arterial dilation actions. Nitroglycerin reduces left ventricular afterload through arterial dilation as well as preload through venous dilation [56,57]. Also the bradycardia caused by systemic b-blockade increases the absolute duration of systole allowing the reflected wave to return sooner to the aorta and to boost central systolic pressure [58]. New antidiabetes drugs, such as incretin-acting drugs and the SGLT2 inhibitors promoting glucosuria. An interesting feature of the incretin drugs is their effect on the cardiovascular system, for example, a lowering of both office and ambulatory BP, a reduction in office BP being observed also following treatment with a SGLT2 inhibitor. This potential vascular protection could be of great importance in patients with features of EVA $[59,60]$. Treatment with statins is possible to reduce EVA in patients with dyslipidaemia.

\section{PERSPECTIVE}

Vascular ageing is one of the most important cardiovascular risk factors. More research is needed into the pathophysiology of premature vascular ageing. This includes detection of the role of molecular mechanisms in the aetiology of vascular stiffness and vascular calcification. It is important to establish non-invasive techniques (including imaging techniques) to study the process of vascular ageing. The development of selective biomarkers for vascular ageing with high predictive value (as opposed to systemic biomarkers, markers of inflammation, circulating bone marrow- derived progenitors, leukocyte telomere length) is an important field of future research. Furthermore, while the role of hypertension is clear, vascular ageing require more research in diabetes mellitus and the metabolic syndrome. The connection between vascular ageing to ageing of other organs such as 
the skin, the kidneys, and the brain is desirable to be studied. It is also necessary to study the interplay between physical activity, nutrition and vascular ageing. Finally, it must be given more importance of the treatment of early vascular ageing independently from all the other cardio-metabolic parameters.

\section{CONFLICT OF INTEREST}

The author(s) confirm that this article content has no conflicts of interest.

\section{ACKNOWLEDGEMENT}

Declared none.

\section{REFERENCES}

[1] Leonard A. The theories of Thomas Sydenham (1624-1689). J R Coll Physicians Lond 1990; 24: 141-3.

[2] Nilsson PM. Early vascular aging (EVA): consequences and prevention. Vasc Health Risk Manag 2008; 4: 547-52.

[3] O'Rourke MF, Safar ME, Dzau V. The Cardiovascular Continuum extended: aging effects on the aorta and microvasculature. Vasc Med 2010; 15: 461-8.

[4] Edward G Lakatta, Daniel Levy. Arterial and cardiac aging: major shareholders in cardiovascular disease enterprises (part I). Circulation 2003; 107: 139-46.

[5] Nilsson PM, Boutouyrie P, Laurent S. Vascular aging: A tale of EVA and ADAM in cardiovascular risk assessment and prevention. Hypertension 2009; 54: 3-10.

[6] Kotsis V, Stabouli S, Karafillis I, et al. Early vascular aging and the role of central blood pressure. J Hypertens 2011; 29: 1847-53.

[7] Reference Values for Arterial Stiffness' Collaboration, et al. Determinants of pulse wave velocity in healthy people and in the presence of cardiovascular risk factors: 'establishing normal and reference values'. Eur Heart J 2010; 31: 2338-50.

[8] Wilkinson IB, Franklin SS, Cockcroft JR. Nitric oxide and the regulation of large artery stiffness: from physiology to pharmacology. Hypertension 2004; 44: 112-16.

[9] Celermajer DS, Sorensen KE, Spiegelhalter DJ, et al. Aging is associated with endothelial dysfunction in healthy men years before the age-related decline in women. J Am Coll Cardiol 1994; 24: 471-6.

[10] Chang E, Harley CB. Telomere length and replicative aging in human vascular tissues. Proc Natl Acad Sci USA 1995; 92: 111904.

[11] Aviv H, Khan MY, Skurnick J, et al. Age dependent aneuploidy and telomere length of the human vascular endothelium. Atherosclerosis 2001; 159: 281-7.

[12] Rauscher FM, Goldschmidt-Clermont PJ, Davis BH, et al. Aging, progenitor cell exhaustion, and atherosclerosis. Circulation 2003; 108: 457-63.

[13] Conboy IM, Conboy MJ, Wagers AJ, et al. Rejuvenation of aged progenitor cells by exposure to a young systemic environment. Nature $2005 ; 433: 760-4$.

[14] Edelberg JM, Reed MJ. Aging and angiogenesis. Front Biosci 2003; 8: s1199-s1209.

[15] Bekaert S, De Meyer T, Rietzschel ER, et al. for the Asklepios Investigators. Telomere length and cardiovascular risk factors in a middle-aged population free of overt cardiovascular disease. Aging Cell 2007; 13: 2960-70

[16] Hansson GK. Inflammation, atherosclerosis and coronary artery disease. N Engl J Med 2005; 352: 1685-95.

[17] Bots ML, Grobbee DE. Intima media thickness as a surrogate marker for generalised atherosclerosis. Cardiovasc Drugs Ther 2002; 16: 341-51.

[18] Stehouwer CD, Henry RM, Ferreira I. Arterial stiffness in diabetes and the metabolic syndrome: a pathway to cardiovascular disease. Diabetologia 2008; 51: 527-39.

[19] Sasaki S, Yoneda Y, Fujita H, et al. Association of blood pressure variability with induction of atherosclerosis in cholesterol-fed rats. Am J Hypertens 1994; 7: 453-9.
[20] Lacolley P, Bezie Y, Girerd X, et al. Aortic distensibility and structural changes in sino-aortic denervated rats. Hypertension 1995; 26: 337-40.

[21] Mancia G, Parati G, Hennig M, et al., ELSA Investigators. Relation between blood pressure variability and carotid artery damage in hypertension: baseline data from the European Lacidipine Study on Atherosclerosis (ELSA). J Hypertens 2001; 19: 1981-9.

[22] Kotsis V, Stabouli S, Karafillis I, et al. Arterial stiffness and $24 \mathrm{~h}$ ambulatory blood pressure monitoring in young healthy volunteers: The early vascular ageing Aristotle University Thessaloniki Study (EVA-ARIS Study). Atherosclerosis 2011; 219: 194-9.

[23] Shan HY, Bai XJ, Chen XM. Apoptosis is involved in the senescence of endothelial cells induced by angiotensin II. Cell Biol Int 2008; 32: 264-70.

[24] Bussemaker E, Hillebrand U, Hausberg M, et al. Pathogenesis of hypertension: interactions among sodium, potassium, and aldosterone. Am J Kidney Dis 2010; 55: 1111-20.

[25] Sansoni P, Vescovini R, Fagnoni F, et al. The immune system in extreme longevity. Exp Gerontol 2008; 43: 61-5.

[26] Salvioli S, Monti D, Lanzarini C, et al. Immune system, cell senescence, aging and longevity: Inflamm-aging reappraised. Curr Pharm Des 2013; 19: 1675-9.

[27] Boutouyrie P, Laurent S, Benetos A, et al. Opposing effects of ageing on distal and proximal large arteries in hypertensives. J Hypertens Suppl 1992; 10: S87-S91.

[28] Vaitkevicius PV, Fleg JL, Engel JH, et al. Effects of age and aerobic capacity on arterial stiffness in healthy adults. Circulation 1993; 88: $1456-62$.

[29] McEniery CM, Yasmin CM, Hall IR, et al. Normal vascular aging: differential effects on wave reflection and aortic pulse wave velocity: the Anglo Cardiff Collaborative Trial (ACCT). J Am Coll Cardiol 2005; 46: 1753-60.

[30] Mitchell GF, Parise H, Benjamin EJ, et al. Changes in arterial stiffness and wave reflection with advancing age in healthy men and women: the Framingham heart study. Hypertension 2004; 43: 1239-45.

[31] Nichols WW, O'Rourke MF. Vascular impedance. In: McDonald's Blood Flow in Arteries: Theoretical, Experimental and Clinical Principles. $4^{\text {th }}$ ed. London, UK: Edward Arnold; 1998: pp. 54-97, 243-283, 347-95.

[32] Safar ME, Frohlich ED. The arterial system in hypertension: a prospective view. Hypertension 1995; 26: 10-4.

[33] Lehmann ED, Gosling RG, Sonksen PH. Arterial wall compliance in diabetes. Diabet Med 1992; 9: 114-9.

[34] Wada T, Kodaira K, Fujishiro K, et al. Correlation of ultrasoundmeasured common carotid artery stiffness with pathological findings. Arterioscler Thromb 1994; 14: 479-82.

[35] London GM, Guerin AP, Marchais SJ, et al. Cardiac and arterial interactions in end-stage renal disease. Kidney Int 1996; 50: 600-8.

[36] Laurent S, Boutouyrie P, Lacolley P. Structural and genetic bases of arterial stiffness. Hypertension 2005; 45: 1050-5.

[37] Tarasov KV, Sanna S, Scuteri A, et al. COL4A1 is associated with arterial stiffness by genome-wide association scan. Circ Cardiovasc Genet 2009; 2: 151-8.

[38] Mitchell GF, Verwoert GC, Tarasov KV, et al. Common genetic variation in the 30-BCL11B gene desert is associated with carotidfemoral pulse wave velocity and excess cardiovascular disease risk: the AortaGen Consortium. Circ Cardiovasc Genet 2012; 5: 81-90.

[39] Ehret GB, Munroe PB, Rice KM, et al. International Consortium for Blood Pressure Genome-Wide Association Studies. Genetic variants in novel pathways influence blood pressure and cardiovascular disease risk. Nature 2011; 478: 103-9.

[40] European Network for Non-invasive Investigation of Large Arteries. Expert consensus document on arterial stiffness: methodological issues and clinical applications. Eur Heart J 2006; 27: 2588605.

[41] Mattace-Raso F, Van der Cammen T, Hofman A, et al. Arterial Stiffness and Risk of Coronary Heart Disease and Stroke: The Rotterdam Study. Circulation 2006; 113; 657-63.

[42] Cruickshank K, Riste L, Anderson S, et al. Gosling Aortic PulseWave Velocity and Its Relationship to Mortality in Diabetes and Glucose Intolerance: An Integrated Index of Vascular Function? Circulation 2002; 106: 2085-90.

[43] Maki-Petaja KM, Elkhawad M, Cheriyan J, et al. Anti-Tumor Necrosis Factor- $a$ Therapy Reduces Aortic Inflammation and Stiff- 
ness in Patients with Rheumatoid Arthritis. Circulation 2012; 126: 2473-80.

[44] Lindharsen J, Ahlehoff O, Gislason GH, et al. The risk of myocardial infarction in rheumatoid arthritis and diabetes mellitus: a Danish nationwide cohort study. Ann Rheum Dis 2011; 70: 929-34.

[45] Harrison DG, Guzik TJ, Lob HE, et al. Inflammation, immunity and hypertension. Hypertension 2011; 57: 132-40.

[46] Asmar RG, Girerd XJ, Brahimi M, et al. Ambulatory blood pressure measurement, smoking and abnormalities of glucose and lipid metabolism in essential hypertension. J Hypertens 1992; 10: 181-7.

[47] Asmar R, Julia PL, Mascarel VL, et al. Ambulatory blood pressure profile after carotid endarterectomy in patients with ischaemic arterial disease. J Hypertens 1994; 12: 697-702.

[48] Staessen J, Amery A, Fagard R. Editorial review. Isolated systolic hypertension. J Hypertens 1990; 8: 393-405.

[49] Safar M. Roles of systolic and pulse pressure. Hypertension 1997; 30: $146-7$.

[50] D'Agostino RB Sr, Vasan RS, Pencina MJ, et al. General cardiovascular risk profile for use in primary care: the Framingham Heart Study. Circulation 2008; 117: 743-53.

[51] Vlachopoulos C, Aznaouridis K, Stefanadis C. Prediction of cardiovascular events and all-cause mortality with arterial stiffness: a systematic review and meta-analysis. J Am Coll Cardiol 2010; 55: 1318-27.

[52] Inoue N, Maeda R, Kawakami $\mathrm{H}$ et al. Aortic pulse wave velocity predicts cardiovascular mortality in middle-aged and elderly japanese men. Circ J 2009; 73: 549-53.
[53] Blacher J, Guerin A, Pannier B, et al. Impact of aortic stiffness on survival in end-stage renal disease. Circulation 1999; 99; 2434-9.

[54] Laurent S, Cockkroft J, Van Bortel L, et al. Expert consensus document on arterial stiffness: methodological issues and clinical applications. Eur Heart J 2006; 27: 2588-605.

[55] Seals DR, Walker AE, Pierce GL, et al. Habitual exercise and vascular ageing. J Physiol 2009; 587: 5541-49.

[56] Kelly RP, Gibbs HH, O'Rourke MF, et al. Nitroglycerine has more favourable effect on left ventricular afterload than apparent from measurement of pressure in a peripheral artery. Eur Heart J 1990; 11: $138-44$.

[57] Duchier J, Iannascoli F, Safar M. Antihypertensive effect of sustainedrelease isosorbide dinitrate for isolated systolic systemic hypertension in the elderly. Am J Cardiol 1987; 60: 99-102.

[58] Wilkinson IB, MacCallum H, Flint L, et al. The influence of heart rate on augmentation index and central arterial pressure in humans. J Physiol 2000; 525: 263-70.

[59] Mistry GC, Maes AL, Lasseter KC, et al. Effect of sitagliptin, a dipeptidyl peptidase-4 inhibitor, on blood pressure in nondiabetic patients with mild to moderate hypertension. J Clin Pharmacol 2008; 48: 592-8.

[60] Paisley AJ, Yadav R, Younis N, et al. Dapagliflozin: a review on efficacy, clinical effectiveness and safety. Expert Opin Investig Drugs 2013; 22: 131-40.

Received: October 15, 2013

Revised: October 18, 2013

Accepted: October 18, 2013

(C) Kotsis et al.; Licensee Bentham Open.

This is an open access article licensed under the terms of the Creative Commons Attribution Non-Commercial License (http://creativecommons.org/licenses/by-nc/3.0/) which permits unrestricted, non-commercial use, distribution and reproduction in any medium, provided the work is properly cited. 